\title{
Comprendre et traiter le narcissisme avec la psychothérapie EMDR
}

\author{
Dolores Mosquera \\ INTRA-TP, Instituto para el estudio del Trauma y los Trastornos de la Personalidad, A Coruña, Espagne \\ Jim Knipe \\ Longmont, Colorado, États-Unis
}

Le trouble de la personnalité narcissique et le trouble de traits narcissiques sont associés à des conduites égoïstes et à un défaut d'empathie envers les autres. Les patients dont la présentation initiale dans la psychothérapie correspond à l'un ou l'autre de ces tableaux ont un profil égocentrique ; ils manquent d'empathie ou se préoccupent peu de la souffrance qu'ils peuvent provoquer chez d'autres personnes, mais ceci n'est qu'un élément parmi d'autres. Parfois le défaut d'empathie et l'égoïsme ne sont que des défenses. Pour appréhender pleinement ce problème, il faut également avoir conscience des difficultés sous-jacentes à se définir soi-même qui sont à l'origine des manifestations comportementales du narcissisme. Comme c'est le cas pour tout problème psychologique, le traitement EMDR nécessite une compréhension de la manière dont les expériences en début de vie conduisent aux symptômes ultérieurs. La compréhension des voies qui relient les expériences vécues à un jeune âge aux traits narcissiques (y compris les présentations latentes) est essentielle à une conceptualisation de cas adéquate, tout comme il faut cerner les structures mentales défensives qui empêchent l'accès aux expériences défavorables fondamentales sous-jacentes aux symptômes.

Mots-clés : narcissisme ; EMDR (désensibilisation et retraitement par les mouvements oculaires) ; trouble de la personnalité narcissique ; trauma ; traitement adaptatif de l'information

es caractéristiques manifestes du narcissisme telles que la grandiosité, la tendance à exploiter les autres, l'arrogance, les problèmes interpersonnels et la rage, sont bien connues des cliniciens, mais les présentations latentes peuvent échapper à toute attention et donc à tout traitement. Le but de cet article est de se centrer sur les diverses formes sous lesquelles le narcissisme peut se manifester, sur la manière de le conceptualiser à partir du modèle du traitement adaptatif de l'information (TAI) et de le traiter avec la psychothérapie EMDR (désensibilisation et retraitement par les mouvements oculaires) (Shapiro, 2001).

\section{Le concept du narcissisme}

Les individus narcissiques peuvent se définir comme des personnes qui sont excessivement préoccupées par des questions d'adéquation personnelle, de pouvoir, de prestige et de vanité (Millon, 1996). Nous pouvons définir le narcissisme à partir de trois points de vue : les comportements d'un individu narcissique, les modalités habituelles de "relations d'objet " du narcissisme (c'est-à-dire, les manières dont une personne narcissique conçoit ses relations avec autrui) et l'aspect phénoménologique du narcissisme (c'est-à-dire, comment est-ce d'être un narcissique ?). Divers aspects

This article originally appeared as Mosquera, D. \& Knipe, J. (2015). Understanding and Treating Narcissism With EMDR Therapy. Journal of EMDR Practice and Research, 9(1), 46-63. Translated by Jenny Ann Rydberg. 
comportementaux de la structure de personnalité narcissique ont été définis en termes de la " triade sombre "- des groupes de traits qui sont associés et coexistent souvent mais qui sont conceptuellement distincts : (a) le narcissisme (sentiment d'ayant-droit, présomption de supériorité aux autres, comportements dominants), (b) le machiavélisme (charme social désinvolte voire éhonté, tendance à la manipulation) et (c) la psychopathie (attitudes sociales froides, impulsivité, antagonisme interpersonnel ; Jonason, Lyons \& Bethell, 2014 ; Kowalski, 2001).

Pour les psychothérapeutes (EMDR) qui traitent des personnes aux traits narcissiques dans le cadre du modèle TAI (Shapiro, 2001), l'aspect phénoménologique est particulièrement important parce qu'une approche TAI cible spécifiquement les émotions non désirées et les expériences subjectives malheureuses des patients. Phénoménologiquement, les personnes narcissiques peuvent se décrire comme excessivement centrées sur et protectrices d'une image de soi hautement valorisée. Cette image est souvent idéalisée, dans le sens où le concept de soi de la personne est faussé, avec une autovalorisation exagérée, des attitudes de singularité, de supériorité, d'ayant-droit et d'immunité à certaines des règles communes qui régissent les conduites appropriées.

En tant que praticiens EMDR, il nous importe de noter que ce genre d'image de soi faussée et excessivement positive, fondée en grande partie sur les expériences de vie antérieures, est susceptible d'être ciblé et résolu à travers des interventions en lien avec l'EMDR. Tout comme les procédures de la psychothérapie EMDR peuvent être utilisées efficacement pour cibler et résoudre les émotions désagréables et une définition de soi excessivement négative, les souvenirs fondamentaux qui engendrent un sens de soi excessivement positif peuvent également être ciblés (Knipe, 2014) pour conduire ces souvenirs à une résolution adaptative avec une autoappréciation plus réaliste.

Pour de nombreux individus, les comportements narcissiques semblent constituer la façade d'un tableau plus complexe. Diverses théories (Gabbard, 1989 ; O. Kernberg, 1975 ; Millon, 1990) postulent que la personne a besoin d'agir comme si elle était supérieure aux autres ou meilleure qu'eux, pour compenser l'autodéfinition interne cachée qui est celle de l'échec ou de la médiocrité.

En plus de ces traits personnels dans le tableau clinique, on trouve aussi souvent un défaut d'empathie vis-à-vis des sentiments et des désirs d'autrui (American Psychiatric Association [APA], 2013), ce que certaines théories expliquent par un déficit au niveau de la "perception d'objets »- la pleine perception de la réalité selon laquelle les autres ont des besoins, perceptions, attitudes et autres caractéristiques distincts (Masterson, 1981). Souvent, les narcissiques ne conçoivent pas les autres comme des êtres humains distincts et différents, mais simplement comme des " objets " à utiliser pour répondre à un besoin personnel (Millon, 1996). Nous émettons l'hypothèse selon laquelle ce déficit d'empathie fait partie des dégâts causés par la frustration en début de vie des "systèmes d'action » d'attachement innés (Van der Hart, Nijenhuis \& Steele, 2006) et le considérons comme une frustration associée aux toutes premières tentatives de se lier avec empathie aux autres, dans la toute petite enfance ou comme une simple imitation de ce qu'ils ont appris de leurs proches (Mosquera, 2012, 2013).

Enfin, l'expérience d'un narcissique peut comporter des moments de plaisir euphorique quand une "victoire » est atteinte, censée prouver sa supériorité. Cependant, nous avons remarqué dans notre pratique clinique que la recherche de ce type de but, même lorsqu'elle réussit, n'a pas d'effet profond. Il est moins satisfaisant d'être admiré en tant que "vainqueur » que d'être véritablement aimé. Dans le traitement d'un patient qui possède des traits narcissiques, ces trois perspectives doivent toutes être prises en compte, comme nous le décrirons dans le texte ci-dessous.

\section{Le développement du narcissisme}

Les études cliniques ont indiqué que les expériences infantiles de maltraitances physiques, sexuelles et émotionnelles, et de négligences physiques et émotionnelles sont fréquentes chez les patients atteints de troubles de la personnalité (Battle et coll., 2004 ; Bierer et coll., 2003 ; D. M. Johnson, Shehan \& Chard, 2004 ; Luntz \& Widom, 1994 ; Rettew et coll., 2003 ; Yen et coll., 2002 ; Zanarini et coll., 2000 ; Zanarini, Gunderson, Marino, Schwartz \& Frankenburg, 1989 ; Zanarini et coll., 1997 ; Zanarini et coll., 2002). Les études impliquant des échantillons de commodité (Gibb, Wheeler, Alloy \& Abramson, 2001 ; Grover et coll., 2007 ; Tyrka, Wyche, Kelly, Price \& Carpenter, 2009) et de petites collectivités (J. G. Johnson, Cohen, Brown, Smailes \& Bernstein, 1999 ; J. G. Johnson, Cohen, Chen, Kasen \& Brook, 2006 ; J. G. Johnson, Smailes, Cohen, Brown \& Bernstein, 2000) ont également soutenu cette association mais il n'est toujours pas clair si la relation entre les troubles de la personnalité et l'adversité dans l'enfance existe dans des échantillons représentatifs de la population générale (Afifi et coll., 2011). Cette recherche investiguait le développement du trouble de la personnalité narcissique mais 
les auteurs supposent que les conclusions de l'étude se rapportent également, peut-être à un degré moindre, aux autres individus qui ne répondent pas aux critères du trouble de la personnalité narcissique mais qui présentent un trouble de traits narcissiques.

Cohen et coll. (2014) rapportent que les maltraitances dans l'enfance, y compris les maltraitances et négligences émotionnelles, physiques et sexuelles représentent un facteur de risque environnemental puissant pour les pathologies de la personnalité. Ces auteurs soulignent qu'un nombre important de recherches établissent le lien entre les maltraitances infantiles et les évolutions défavorables à l'âge adulte, incluant les pathologies de la personnalité (Collishaw et coll., 2007 ; Lobbestael, Arntz \& Berstein, 2010). Toutefois il n'est toujours pas clair si certains types donnés de maltraitances dans l'enfance prédisent des types spécifiques de pathologie de la personnalité.

Des études méta-analytiques et empiriques suggèrent que les traits de personnalité narcissiques egocentriques augmentent à travers le monde depuis quelques décennies (Cai, Kwan \& Sedikides, 2012; Twenge, Konrath, Foster, Campbell \& Bushman, 2008 ; toutefois, voir aussi Trzesniewski, Donnellan \& Robins, 2008). Cette "épidémie de narcissisme " s'observe à travers l'augmentation des scores sur l'inventaire de la personnalité narcissique (Narcissistic Personality Inventory ; Twenge et coll., 2008), le contenu des paroles des chansons pop (DeWall, Pond, Campbell \& Twenge, 2011), l'usage égocentrique de mots et de phrases dans les livres (Twenge, Campbell \& Gentile, 2012) et la diminution de la propension à l'empathie (Konrath, O'Brien \& Hsing, 2011). Le narcissisme a été associé aux caractéristiques d'une culture (Foster, Campbell \& Twenge, 2003) et le changement sur le plan de la propension à l'empathie pourrait avoir un lien direct avec le changement culturel (Cai et coll., 2012) qui est souvent entraîné par le développement économique (Hamamura, 2012). Malgré la prolifération des recherches sur les différences générationnelles et culturelles concernant le narcissisme, les causes immédiates de ces différences ne sont toujours pas claires (Lyons, Morgan, Thomas \& Al Hashmi, 2013).

\section{Le narcissisme et les modèles psychothérapeutiques de I'EMDR/ traitement adaptatif de l'information et de la psychanalyse}

Une approche EMDR du traitement du narcissisme commence par la supposition - une hypothèse - que le narcissisme se fonde sur des expériences de vie à un jeune âge, difficiles et dommageables, soit des événements traumatiques distincts particuliers (Afifi et coll., 2011), soit la situation plus fréquente des «traumatismes par omission " - la situation dans laquelle un enfant est privé de manière répétée au niveau de ses besoins d'attachement et d'éducation (Cohen et coll., 2014). Nous présumons que, chez l'individu adulte atteint de narcissisme, des traumas des deux sortes ont eu une influence sur la création non seulement de souvenirs stockés de manière dysfonctionnelle mais aussi d'autres éléments fondés sur le trauma et enregistrés de manière dysfonctionnelle (Gonzalez \& Mosquera, 2012) tels que des défenses psychologiques et souvent la dissociation (autrement dit, un défaut de plein accès conscient entre des états de soi/réseaux mnésiques fonctionnant séparément).

Les fondements théoriques de notre travail correspondent à une intégration du modèle TAI de l'EMDR (Shapiro, 2001) avec une perspective psychanalytique (O. Kernberg, 1975 ; Kohut, 1971 ; Masterson, 1981 ; Millon, 1996). Ces modèles divergent des manières suivantes : la théorie psychanalytique a l'inconscient, les projections et les défenses. Les défenses sont conçues comme servant une fonction protectrice et elles s'apprennent dans la petite enfance comme un moyen de compenser les échecs ou le défaut d'accordage. Le modèle thérapeutique TAI, par contre, voit les fondements de la psychopathologie dans des réseaux mnésiques dysfonctionnels et postule que certains réseaux sont maintenus hors du champ de la conscience parce que l'activation de tels réseaux pourrait s'avérer submergeante et menaçante. Ces modèles thérapeutiques partagent la supposition selon laquelle les expériences de la petite enfance peuvent être en lien avec des caractéristiques particulières de la psychopathologie adulte.

Les deux théories envisagent les traits narcissiques comme les résultats finaux d'un environnement négligeant, de maltraitances chroniques ou d'autres expériences défavorables (Afifi et coll., 2011) mais ces traits peuvent aussi se trouver en lien avec une appréciation irréaliste et un dorlotage excessif (Jonason et coll., 2014 ; Kowalski, 2001 ; Millon, 1990 ; Stone, 1993). Diverses perturbations de l'attachement aux parents ou à leurs substituts peuvent également conduire à un défaut d'empathie et à l'égocentrisme (Bennett, 2006 ; Campbell \& Foster, 2002 ; Cramer, 2010 ; P. F. Kernberg, 1998 ; Lyons et coll., 2013). Puisque le narcissisme fonctionne souvent comme une défense psychologique (P. F. Kernberg, 1998 ; Knipe, 1998 ; Mosquera, 2008), c'est-à-dire comme une façon d'empêcher la conscience ou l'émergence d'une perturbation émotionnelle, toutes sortes de 
circonstances de vie difficiles peuvent conduire au développement de cette défense.

Dans cet article, nous proposons l'hypothèse selon laquelle, avec une préparation adéquate et un accordage et une compassion appropriés de la part du psychothérapeute, tant les souvenirs dysfonctionnels que les autres éléments stockés de manière dysfonctionnelle peuvent être amenés à une résolution adaptative grâce à des interventions TAI particulières, comprenant des séries focalisées de stimulations bilatérales. Cet article apporte des lignes directrices générales et ne prétend pas minimiser l'énorme complexité et variété des structures de personnalité narcissiques qui peuvent sous-tendre la présentation comportementale manifeste.

\section{Le narcissisme et le trauma en début de vie}

Les psychothérapeutes connaissent souvent le narcissisme d'abord en tant que cause de souffrance ou de dégâts, occasionnés par des individus narcissiques, chez les patients qui viennent en psychothérapie. Pour les patients à qui on a fait du mal de cette façon, il faut se centrer sur la manière dont les maltraitances ont provoqué des émotions et une autodéfinition de victime impuissante. Mais quand le patient est un individu qui possède des traits narcissiques, une perspective différente est nécessaire, prenant en compte l'expérience qu'a cet individu de lui-même et des autres, avec une conscience chez le psychothérapeute de la manière dont un style narcissique peut empêcher cette personne d'apprécier normalement les expériences de vie satisfaisantes, d'atteindre les buts psychothérapeutiques et d'avoir des relations saines avec autrui.

On pourrait concevoir le narcissisme comme un problème émotionnel qui est souvent bien caché des autres et évident seulement dans ses effets délétères sur l'individu lui-même ou sur les personnes avec lesquels il est en relation. Les personnes qui sont narcissiques consacrent beaucoup d'énergie non seulement à s'adapter mais aussi à paraître exemplaires, meilleures que les autres. La plupart des individus seraient plus ou moins désemparés dans les moments où ils apparaissent comme maladroits ou imparfaits aux yeux des autres, mais pour la personne narcissique, cette détresse se trouve probablement amplifiée parce qu'elle constitue la perte d'une défense importante contre des sentiments perturbants non encore résolus. Par exemple, si l'individu narcissique a appris, enfant, que " je dois paraître intelligent et impressionnant auprès des autres en tout temps pour mériter l'amour de mes parents ", alors même une défaillance très mineure au niveau de l'apparente intelligence pourrait faire surgir tous les sentiments d'abandon émotionnel qui se sont produits dans la relation dysfonctionnelle originelle avec les parents. Ainsi l'individu est contraint à entretenir une apparence impressionnante en tout temps afin de maintenir cette défense et d'éviter une réactivation de ces sentiments de la petite enfance d'être abandonné et indigne d'amour (P. F. Kernberg, 1998). Pour toutes ces raisons, le traitement du narcissisme en EMDR impliquera probablement le ciblage de traumatismes d'omission en début de vie, ces moments où l'indifférence des parents envers l'enfant pouvait l'influencer dans le sens de devenir la réplique presque parfaite de ce dont les parents avaient besoin. De cette manière, le besoin de connexion de l'enfant pouvait être partiellement satisfait (Cramer, 2010 ; Winnicott, 1965).

Cela ne veut pas dire que le narcissisme est entièrement causé par des expériences difficiles en début de vie. Le narcissisme est un trait de personnalité partiellement en lien avec des facteurs génétiques (Livesley, Jang, Jackson \& Vernon, 1993). Néanmoins, une hypothèse centrale de l'approche TAI présentée ici et ailleurs (Stone, 1993) est que les expériences défavorables de l'enfance et les perturbations de l'attachement contribuent considérablement au problème du narcissisme chez l'adulte, particulièrement en ce qui concerne le défaut d'empathie et l'égocentrisme.

\section{L'égocentrisme pathologique et le narcissisme normal dans l'enfance}

Les enfants présentant une pathologie narcissique manifestent une sorte d'égocentrisme qui diffère du narcissisme normal de l'enfance (P. F. Kernberg, Weiner \& Bardenstein, 2000). Dans le narcissisme normal, le besoin de dépendance et d'admiration chez l'enfant est assouvi par l'attention reçue de manière adaptée selon l'âge ; il est capable de reconnaître ce soutien attentionné avec réciprocité et gratitude (P. F. Kernberg et coll., 2000) et d'apprendre aisément la satisfaction apportée par l'empathie et l'implication auprès des autres. Le développement normal peut être freiné en cas de perturbations de l'attachement (Bennett, 2006) et de diverses expériences défavorables (Afifi et coll., 2011 ; Lyons et coll., 2013), y compris le fait de ne pas être vu par les proches avec un regard à la fois réaliste et aimant (Knipe, 2007 ; Millon, 1990 ; Mosquera \& Gonzalez, 2011 ; Stone, 1993). Le chemin depuis les expériences de début de vie jusqu'au développement de traits narcissiques peut varier et se tracer différemment chez différents patients. Selon notre point de vue, le soi narcissique faussé se comprend comme une 
défense contre des souvenirs traumatiques, généralement des traumas d'omission et d'absence d'implication, mais parfois aussi des traumas de maltraitances manifestes.

\section{Une recherche désespérée de considération positive}

Des expériences traumatiques très diverses peuvent sous-tendre la présentation narcissique apparente. Les auteurs observent que le schéma est établi très tôt, généralement dans le cadre de la manière dont l'enfant est traité par des proches différents, ou traité différemment d'une fois à l'autre par la même personne. Un enfant peut acquérir des concepts de soi différents de la part de différents proches - par exemple, lorsqu'un enfant est idéalisé par un parent et fortement critiqué par l'autre. Ceci peut se produire quand la mère gâte et que le père est critique, ou inversément, et que les parents se polarisent dans leurs réactions à l'enfant. C'est très déroutant pour l'enfant, et dans ces cas, les parents utilisent souvent l'enfant comme le champ de bataille de leurs propres conflits irrésolus. Le schéma de deux états du moi séparés peut aussi s'établir quand aucun des parents ne renvoit suffisamment en miroir ni ne reconnaît l'expérience interne de l'enfant qui cherche désespérément une façon de recevoir une considération positive de la part d'un ou des deux parents. Tenter d'être parfait aux yeux des autres n'est pas vraiment épanouissant en termes de connexion mais peut devenir une " action substitutive " (Van der Hart et coll., 2006), un comportement avec un haut degré de motivation dans le but de ressentir ne fût-ce qu'un petit peu de connexion.

\section{Apprendre la valeur de la supériorité}

Les parents de l'enfant peuvent avoir de traits narcissiques qui sont dissimulés des personnes extérieures à la famille mais qui sont connus et imités par leurs enfants (Mosquera, 2012). L'environnement familial peut paraitre " normal » en surface mais à niveau plus profond, on peut trouver un accordage, une connexion, une affection et une reconnaissance des besoins de l'enfant insuffisants (Afifi et coll., 2011 ; Bennett, 2006 ; Cramer, 2010 ; P. F. Kernberg, 1998). Nous avons vu des patients qui ont grandi dans de telles circonstances et qui ont appris qu'être unique et supérieur constituait le moyen de gagner de l'acceptation et donc de renforcer la fragile connexion aux proches. Les aspects de l'enfant qui étaient «non exceptionnels » devaient être cachés, peut-être même écartés de la conscience de l'enfant. Un patient a décrit comment, à l'âge de sept ans, il s'est senti très positivement lié à ses parents quand il a rejoint leur soirée cocktail et a fait le tour des invités, leur serrant la main et tentant de prononcer des mots d'esprit à chacun. Quand, dans le cadre du traitement, ce souvenir initialement positif a été ciblé, ses pensées sont rapidement passées à : «Je devais impressionner tout le monde, mais je n'avais qu'une hâte, c'était de sortir de là ! ”En d'autres termes, ses sentiments positifs n'étaient pas très profonds et n'étaient en réalité qu'une pellicule sur ses sentiments plus fondamentaux d'insuffisance et de manque de connexion avec ses parents égocentriques. Dans sa vie adulte, ce patient s'était fait « un million d'amis » mais il était frustré par le manque de véritable intimité. Il lui était utile d'apercevoir les origines de cette façon particulière d'entrer en relation avec les autres, afin qu'il puisse ensuite avancer pour apprendre comment atteindre une proximité plus réelle et plus satisfaisante avec les autres.

Même lorsque les proches de l'enfant ne sont pas manifestement narcissiques, ils peuvent se servir de l'enfant pour compenser leurs désirs frustrés ou leur croyance d'être inférieurs et sans valeur (P. F. Kernberg, 1998 ; Millon, 1990 ; Segrin, Woszidlo, Givertz \& Montgomery, 2013 ; Stone, 1993). On pense que les enfants qui grandissent dans une famille insuffisamment soutenante évolueront vers une propension à répondre aux besoins des parents comme moyen de maintenir une connexion nécessaire avec ces proches. Nous avons observé que quand le parent a une personnalité narcissique, même dissimulée, le principal moyen de connexion avec lui peut consister à devenir son " objet narcissique », un enfant qui semble idéal. Une connexion parent-enfant est ainsi établie, mais le prix à payer pour l'enfant est le désaveu de ses besoins et de ses qualités intrinsèques et particuliers que le parent ne peut pas reconnaitre ou tolérer. Cette situation peut se trouver exacerbée si l'enfant devient l'objet de félicitations et de dorlotage excessifs et irréalistes, ainsi que d'attentes positives exagérées (que l'enfant soit exceptionnellement doué ou pas), ce qui peut engendrer ce que Tartakoff (1966) appelait le complexe du prix Nobel. Les sentiments de supériorité et d'être destiné à accomplir de grandes choses peuvent être exaltants à contempler pour l'enfant mais également une source de pression émotionnelle énorme et en décalage avec les capacités ou comportements réels de l'enfant.

\section{Le développement d'un sentiment d'importance grandiose}

Certains patients avec des tendances narcissiques grandissent avec un sens de soi faussé qui reflète les distorsions au niveau de la manière dont leurs proches 
les traitaient. Dans certains cas, nous avons vu des parents « apparemment soutenants » qui encouragaient des attitudes d'ayant-droit. Ces parents n'encourageaient pas les attitudes appropriées d'humilité et d'introspection - c'est-à-dire comment apprendre de ses erreurs tout en maintenant un sentiment de valeur personnelle.

Comme le déclarait Stone (1993), le narcissisme peut provenir tant du " trop » (surprotection, compliments excessifs) que du " pas assez » (environnements gravement traumatisants ou négligeants), et les deux conditions peuvent se produire au sein de la même famille. Stone parle de compensation pour décrirer cette structure de personnalité car le sentiment d'être quelqu'un d'exceptionnel a pour fonction de compenser, au moins en partie, l'indifférence parentale et les négligences. Dans cette situation, l'enfant peut développer un désir exagéré de "grandeur » pour développer un sentiment de valeur personnelle en l'absence de compliments parentaux plus ordinaires et ancrés dans la réalité. Dans ce cas, l'expérience interne et les comportements externes de l'enfant négligé peuvent exprimer deux concepts de soi très différents : un sentiment extérieur d'exception compensatoire qui cache un sentiment intérieur d'autodépréciation (Stone, 1993). On pense que les traits narcissiques fonctionnent dans ce contexte comme des défenses qui empêchent l'émergence dans la conscience de sentiments d'autodévalorisation (Nathanson, 1992) qui peuvent être considérés comme un symptôme post-traumatique trouvant son origine dans le trauma d'un abandon émotionnel répété. Ces défenses sont souvent complexes et élaborées (P. F. Kernberg, 1998).

\section{Le développement d'une grande vulnérabilité face aux avis négatifs des autres}

Bien qu'un sentiment d'importance grandiose soit central dans le trouble de la personnalité narcissique, la vulnérabilité de l'estime de soi en est un trait associé (P. F. Kernberg, 1998 ; P. F. Kernberg et coll., 2000 ; Millon, 1990). Étant donné que l'image de soi idéalisée du patient narcissique est une distortion de ce qui est réellement vrai et qu'il est fortement investi dans le maintien de cette image, la perception de la réalité elle-même peut être très menaçante et les personnes qui disent librement la vérité peuvent être vues comme des menaces. Volkan (1973) a décrit les diverses manœuvres défensives (c.-à-d. rejeter la faute, dévaloriser) utilisées par les individus narcissiques pour protéger le soi grandiose de l'assaut de la réalité. Ainsi, la structure de la personnalité narcissique peut se développer pour permettre la connexion avec les proches, pour se défendre contre des sentiments d'abandon post-traumatiques, mais aussi en tant qu'intériorisation des proches narcissiques inadéquats.

Le sens de soi grandiose de l'enfant peut se comprendre comme résultant de la fusion des aspects positifs du soi réel, du soi idéal et des modèles idéaux, avec l'élimination de tout ce qui serait incompatible (qui est partiellement ou complètement dissocié), donc ce qui reste est une image de soi exagérément positive (P. F. Kernberg et coll., 2000). Une personne aux traits narcissiques doit désavouer tout ce qui ne correspond pas à son sens de soi grandiose, ce qui implique de percevoir les autres (et soi-même) de manière déformée. Les autres, ainsi que certaines parties du soi, sont dévalorisés et séparés, entraînant un défaut d'intégration. Cette image de soi grandiose est nécessaire pour prévenir tout contact avec une vulnérabilité intérieure douloureuse. À mesure que l'enfant grandit, le prix à payer pour ressentir la connexion consiste donc à continuer à être l'incarnation parfaite de l'image qu'ont les parents de "l'enfant parfait». L'enfant peut alors généraliser depuis les parents à toutes les personnes et agir comme si la perfection était nécessaire pour gagner l'acceptation des autres : «Je dois être parfait pour être bien a minima ». Une supposition sous-jacente, pas toujours consciente mais fortement présente dans les croyances de la personne sur les relations, va comme suit : "Si des personnes ne sont pas d'accord avec mon sens de soi très positif, alors elles m'attaquent ! » En entrant dans la vie adulte, cette attitude suscitera une forte réactivité aux critiques d'autrui (tout ce qui renvoie à la personne qu'elle n'est pas parfaite) et les autres auront souvent l'impression qu'elle essaie de convaincre tout le monde de sa perfection. Les autres évidemment n'apprécient guère cette façon de faire et tendent à s'écarter, ce qui réveille les souvenirs d'abandon et d'indifférence de l'individu narcissique (Shapiro, 2001), restimulant le besoin d'être perçu comme parfait aux yeux des autres (P. F. Kernberg et coll., 2000). Nous faisons l'hypothèse qu'il s'agissait, dans l'enfance, de la seule défense disponible contre l'abandon total.

\section{Présentations du narcissisme dans le traitement}

La présentation apparente d'un patient aux traits narcissiques ne représente pas toujours le tableau complet de toute la structure de la personnalité. La présentation apparente est souvent une armure qui recouvre un soi extrêmement vulnérable. Cet état du soi vulnérable peut être partiellement ou complètement indisponible à un accès conscient. Les nouveaux critères 
du DSM-5 (Diagnostic and Statistical Manual of Mental Disorders, $5^{\mathrm{e}}$ édition ; APA, 2013) tentent de refléter cette ambivalence, expliquant que l'autoappréciation peut être positivement ou négativement exagérée, ou vaciller entre des extrêmes. En d'autres termes, les traits narcissiques, bien que dommageables tant pour l'individu que pour les autres, ne sont pas nécessairement présents à tout moment au niveau des comportements de l'individu ; ceci peut être déroutant pour les patients, leurs partenaires de vie et leurs psychothérapeutes.

Gabbard (1989) affirme que la description habituelle du trouble de la personnalité narcissique se centre sur les caractéristiques " manifestes » du narcissisme (grandiosité, exploitation, arrogance, problèmes interpersonnels et rage) en omettant celles, « latentes ", qui sont moins évidentes et plus subtiles (tendance à être sensible à la honte, introverti, vulnérable, inhibé et porté à l'anxiété). Bien que de nombreuses présentations narcissiques ne soient que la couverture d'un soi vulnérable, il faut éviter les généralisations. Il existe un groupe de patients avec une pathologie narcissique sévère qui ont complètement et fortement dissocié tous les souvenirs d'enfance d'abandon et de maltraitance et qui par conséquent n'ont pas tendance à se sentir « mal » ou « inférieurs » ni à avoir une expérience interne de vide ou de creux. Au contraire, ils peuvent essayer d'utiliser la psychothérapie pour manipuler leurs proches. Un psychothérapeute bien intentionné peut poser trop de questions orientées et percevoir à tort un faux "soi vulnérable " et des souvenirs traumatiques inexistants de la petite enfance, en manquant d'insister suffisamment sur la protection des autres, tels que les membres de la famille, qui pourraient se trouver en danger (Mosquera, 2012).

\section{Le « narcissique furtif»}

Il est également possible que les traits narcissiques ne deviennent apparents dans le traitement qu'après un certain délai. Masterson (1981) définit le " narcissique furtif " comme un patient qui parait humble, farouche, timide, inhibé ou inefficace, pour ne révéler que plus tard dans la psychothérapie les fantasmes les plus élaborés d’un soi grandiose.

Le « narcissique sans scrupule »

Dans des cas de maltraitances évidentes, conduisant à des procédures judiciaires, certains patients aux tendances narcissiques sont orientés en psychothérapie. D’autres fois, ils sont amenés par les mêmes proches (conjoint, parents) qui ont subi les conséquences de leur caractère et qui sont maintenant forcés à se faire soigner. Souvent, ce n'est pas la personne à la personnalité narcissique qui arrive en tant que patient. Elle arrive plutôt en tant que proche "coopératif " d'une personne qui demande une psychothérapie en raison de divers symptômes, alors que bon nombre de ces symptômes ont été provoqués par l'exigence et les attentes égoïstes de cette personne.

Dans les cas de personnes qui ont encore moins d'empathie envers les autres et qui peuvent se décrire comme des individus antisociaux (tels que le " narcissique sans scrupule »), la psychothérapie sera sans doute très difficile. Le comportement du narcissique sans scrupule se caractérise par un sentiment arrogant de valeur personnelle, une indifférence quant au bienêtre des autres ainsi qu'un comportement en société fourbe et intimidant. Ces individus sont conscients d'exploiter les autres et s'attendent à une reconnaissance et à une considération particulières sans assumer de responsabilité réciproque. Ils n'ont pas de véritable sentiment de culpabilité et peu de conscience sociale. Ils entretiennent leurs relations tant qu' ils ont quelque chose à en tirer (Millon, 1996).

Dans certains cas, ils éprouvent du plaisir faire du mal aux autres (Mosquera, 2007). Nous pouvons envisager ces personnes comme des individus qui ont appris très tôt dans la vie à éviter l'attachement parce qu'il était toujours associé à une douleur émotionnelle. Elles sont difficiles à traiter parce que leur défense de dévalorisation est si profondément ancrée qu'elle est devenue leur identité. Souvent, dans ces cas-là, une intervention psychothérapeutique plus réaliste sera de les aider à connaître les conséquences négatives de leurs comportements ou de protéger les autres de ces individus.

\section{Le « narcissique élitiste »}

Certains individus à la personnalité narcissique présentent un profil plus subtil, celui du " narcissique élitiste " (Millon, 1996), initialement décrit par Reich (1949) en tant que " narcissique phallique ». Parfois ils ressemblent à des personnes bien adaptées, mais comme ils parlent excessivement d'eux-mêmes, ils sont sujets à des discordances entre ce qu'ils sont et la manière dont ils se présentent. Contrairement à beaucoup d'autres qui sont conscients de cette disparité, les « élitistes " sont entièrement convaincus d'être formidables. Plutôt que de faire l'effort d'acquérir de véritables qualifications et du talent, ils consacrent beaucoup d'énergie à persuader les autres de leur caractère exceptionnel. Ils peuvent être choqués, déçus et même fâchés quand les autres ne leur confirment pas leur image de soi hypervalorisée. Ce sont souvent 
des arrivistes qui essaient de cultiver leur sentiment d'être exceptionnels et leurs bénéfices personnels en s'associant à des personnes qui connaissent de véritables accomplissements et une réelle reconnaissance de la part d'autrui. Quelle que soit l'activité entreprise, ils consacrent leur énergie au fait de se promouvoir, en vantant leurs réalisations, vraies ou fausses, en tenant de persuader que tout ce qu'ils ont fait est extraordinaire, mieux que tout ce qu'ont pu faire les autres, dépassant de loin la réalité (Millon, 1996). Dans ces cas, il peut y avoir une histoire considérable d'ostentation, de se trouver au centre de l'attention, de symptômes exagérés, de grande importance et du désir d'être différent. Ces patients considèrent qu'ils doivent être traités différemment parce qu'ils sont exceptionnels. Derrière cette attitude, il existe souvent une fragmentation de l'identité véritable ou une difficulté extrême à se relier émotionnellement. Un exemple en est un homme d'âge mûr qui s'est senti émotionnellement abattu lorsque son épouse, après des années pendant lesquelles elle s'est sentie ignorée, critiquée et humiliée par son mari, a demandé le divorce.

\section{Le « narcissique victime »}

Le fait de se définir soi-même en tant que "victime " peut se substituer à une identité plus complète et réaliste. L'histoire d'une telle personne peut comprendre des négligences émotionnelles graves dans la petite enfance, mais dans leur vie actuelle, elle s'attend à ce que les autres répondent à ses besoins et lui donnent ce qu'elle n'a jamais eu. De nombreuses personnalités aux traits narcissiques finissent par construire une identité autour de l'idée que «tout le mal qui existe m’est arrivé ». Ce type de raisonnement et cette manière de " voir la vie » (à travers un filtre particulièrement négatif et autoréférencé) génère généralement une grande souffrance et beaucoup de difficultés d'adaptation (Mosquera, 2008). Le sentiment de la personne d'avoir été une victime est, dans un certain sens, juste en ce qui concerne les maltraitances infantiles, mais ce sentiment d'être une victime est ensuite appliqué à tort à de nombreuses situations présentes, causant des réactions négatives à répétition chez les autres. L'aspect narcissique de cette présentation clinique consiste en un sentiment d'ayant-droit : «J'ai été maltraité dans le passé, donc aujourd’hui, je mérite un traitement d'exception. » De cette manière, le schéma se répète ; l'identité de victime conduit paradoxalement à une de nouvelles maltraitances car le patient ne peut continuer à jouer ce rôle (qui est ressenti comme familier et essentiel à la survie psychologique) qu'à travers des relations maltraitantes.
Une variante de ce sous-type de la personnalité correspond à une personne qui s'accroche à une étiquette diagnostique en tant qu'identité substitutive.

Il s'agit d'une autoévaluation en tant que " cas difficile et particulier ». Par exemple, un professeur d'univerité de 52 ans a demandé un rendez-vous en déclarant que la psychothérapeute serait chanceuse de pouvoir l'évaluer car il était le cas le plus sévère qu'elle verrait de sa vie. Une telle attitude peut être un « appel à l'aide » mais cela peut également signaler de manière diagnostique au psychothérapeute que le patient pourrait avoir un fort sentiment d'ayant-droit et l'impression qu'il serait injuste d'être tenu à des attentes normales en termes de responsabilité.

\section{Les patients « oui, mais »}

Certains patients peuvent se présenter avec une estime de soi apparemment faible et une dépression, ce qui mobilise généralement l'attention et le soutien des autres. Cependant, cette attention et ce soutien ne semblent jamais suffire ; c'est comme s'il leur fallait « quelque chose de plus », quelque chose qu'ils ne peuvent trouver mais qui pourrait finir par les combler. Ces individus demandent de l'aide, réclament un traitement et viennent aux rendez-vous mais manifestent une forte ambivalence quant à l'aide reçue et tendent à faire le contraire des propositions qu'ils semblent initialement accepter (Mosquera, 2008). À un niveau de communication pragmatique, ces verbalisations négatives à leur propre sujet constituent une tentative de susciter des commentaires de soutien de la part des autres. Au fond d'eux, leurs croyances sont " je suis supérieur aux autres", "mes valeurs sont supérieures » et «le monde est injuste et je suis une pauvre victime ». Ils attribuent généralement leurs problèmes à une cause extérieure et éprouvent d'importantes difficultés à assumer leurs responsabilités ou à se centrer sur ce qui dépend d'eux. Parfois leur style est passif-agressif, incitant les autres à faire des choses pour eux, pour ensuite leur donner un sentiment d'inutilité car rien n'est jamais assez.

\section{Le soignant infatigable ou « narcissique qui fait plaisir»}

Certains patients sont concentrés sur le fait d'obtenir l'approbation des autres et sont très vulnérables aux critiques. Tous les comportements sont destinés à montrer à autrui l'image d'une "personne extrêmement bonne »; ces individus semblent vivre " pour » les autres et ne comprennent pas pourquoi les autres « ne le leur rendent pas ». Bien qu’ils semblent apprécier " faire plaisir » aux autres, ils en attendent en 
réalité quelque chose en retour mais ils ont du mal à le reconnaître et éprouvent colère et frustration quand ces personnes ne réagissent comme ils s'y attendent. Ils peuvent développer une façade élaborée de «bonté » et sont déroutés par les réactions négatives des autres (Mosquera, 2008). Il peut y avoir une certaine grandiosité dans leur présomption de responsabilité vis-àvis des sentiments et comportements de tous ceux qui les entourent. Ce type d'individu peut souffrir d'une "blessure narcissique " ou éprouver une colère indignée quand les autres ne réagissent pas comme prévu à sa bonté. Les patients de ce sous-type peuvent être source de traumatisation pour leurs enfants qui les décriront souvent à l'âge adulte comme des " parents formidables " tout en se sentant confus et coupables d'éprouver du ressentiment envers ces parents dévoués, dans l’abnégation, mais égocentriques.

\section{Les difficultés du travail en EMDR avec le patient atteint de narcissisme}

Pour les psychothérapeutes qui traitent le narcissisme, il peut être difficile de gérer des émotions contre-transférentielles négatives envers des patients qui évitent de manière phobique de laisser le psychothérapeute compter et qui ont une réaction instinctive de rejeter la faute sur les autres dès qu'ils rencontrent des frustrations dans leur vie. Ces patients peuvent dire des choses qui placent le psychothérapeute sur la défensive, comme " qu'est-ce qui vous fait croire que vous pouvez m'aider? » ou, comme l'a dit un patient, " mon principal problème, c'est que les autres ne se comportent pas comme ils le devraient. Comment l'EMDR pourrait-il y changer quelque chose ? ". Le psychothérapeute peut entamer une conversation sur les diverses options possibles lorsque les autres " ne se comportent pas comme ils le devraient " et le but de la psychothérapie peut consister à avancer vers l'une d'elles (par exemple, l'acceptation ou une conversation constructive orientée vers la résolution de problèmes).

Il est évident que ce schéma de personnalité peut initialement empêcher le patient de s'engager dans un traitement EMDR réel de ses expériences de vie traumatiques. La posture de la pleine conscience proposée par Shapiro (2001) en tant que prérequis à l'accès et au traitement d'un souvenir n'est généralement pas présente dans les troubles de la personnalité, dans lesquels les perturbations précoces de l'attachement n'ont pas permis le plein développement de l'intégration entre les niveaux de traitement cognitif, émotionnel et sensorimoteur. Par conséquent, la personne a développé des structures mentales défensives pour empêcher le contact insupportable avec la perturbation et la vulnérabilité (Afifi et coll., 2011 ; Bennett, 2006).

\section{L'activation des structures mentales défensives}

Pour le patient aux tendances narcissiques, ces structures mentales défensives tendent à s'activer rapidement et automatiquement en réponse à toute menace au soi faussé idéalisé. Dans nos pratiques, nous avons vu que les clients atteints de narcissisme ont du mal à considérer les problèmes conflictuels avec les autres avec réalisme et à identifier leur propre contribution à ces problèmes. Ils sont plutôt susceptibles de rejeter la faute sur les autres, rapidement et presqu'automatiquement, apparemment pour protéger leur image de soi idéalisée.

\section{Le narcissisme latent}

Une structure narcissique de la personnalité peut être très manifeste et évidente pour de nombreuses personnes qui observent l'individu atteint de narcissisme, ou bien latente et bien cachée, sauf à ceux qui essaient d'avoir une relation intime avec l'individu. Comment le psychothérapeute peut-il détecter le narcissisme latent? Les traits narcissiques manifestes sont facilement identifiés, mais les tableaux latents le sont plus difficilement. Une structure narcissique cachée de la personnalité deviendra généralement plus évidente dans la réaction excessive du patient à une critique ou à la révélation d'un défaut qui est, objectivement, mineur. Les patients narcissiques non détectés peuvent essayer (parce que c'est fréquent dans leur schéma de fonctionnement) d'attirer l'attention et l'admiration du psychothérapeute en jouant le rôle qu'ils pensent être celui que leur thérapeute préférerait. De plus, ils peuvent décrire des problèmes et des souvenirs aisément résolus pour renforcer la connexion avec le psychothérapeute.

\section{L'identification projective}

Les psychothérapeutes travaillant avec des patients qui ont des traits narcissiques devraient rester attentifs à un certain type d'identification projective dans lequel le psychothérapeute est idéalisé par le patient, puis le thérapeute incorpore la projection idéalisée du patient et commence à l'agir en tant que contre-transfert. Le sentiment du patient dans ces séances pourrait être « je suis quelqu'un d'unique et de supérieur et vous, vous êtes mon psychothérapeute unique et supérieur ». Il peut s'agir d'un piège qui fait échouer la psychothérapie si le thérapeute s'investit dans cette image de soi idéalisée ou si le patient, à cause de l'idéalisation mutuelle, hésite à 
révéler ses défaillances personnelles. Ce schéma d'identification projective peut s'être déjà produit à de nombreuses occasions dans les autres relations du patient. Pour le patient atteint de narcissisme, les " 10 événements les plus perturbants " peuvent être des situations dans lesquelles les autres n'ont pas confirmé le soi idéalisé du patient ou dans lesquelles cet idéal n'était plus tenable d'une façon ou d'une autre - quelque chose s'étant produit qui a révélé que le client n'était pas si supérieur que cela.

\section{Un traitement long}

À cause de la complexité des personnalités narcissiques, un long processus de démantèlement de la structure de personnalité dysfonctionnelle sera généralement nécessaire (Mosquera, 2012). Le psychothérapeute doit accompagner ce démantèlement d'un haut niveau d'accordage et d'attention à la sécurité émotionnelle du patient. Nous enseignons souvent à nos patients atteints de narcissisme comment accepter la réalité de l'accordage juste d'une autre personne - quelque chose que le patient n'a pas suffisamment reçu dans son enfance. Le processus psychothérapeutique est souvent nécessairement long parce que sous l'apparence narcissique, on peut trouver une réactivité extrême et une vulnérabilité aux sentiments d'abandon ou d'être injustement attaqué ou humilié.

\section{Quand et pourquoi des individus atteints de} narcissisme viennent-ils en psychothérapie?

Les personnes qui possèdent des caractéristiques ou des traits narcissiques peuvent éprouver un malheur énorme dans leur vie mais elles sont souvent récalcitrantes à l'idée de suivre une psychothérapie. Il peut $\mathrm{y}$ avoir de la peur de se trouver dans une situation d'évaluation vis-à-vis d'une autre personne, de devoir révéler des informations personnelles qui pourraient s'écarter de leur image de soi idéalisée, ou dans une situation où le fait de raconter la vérité sans censure constitue la norme. Les patients atteints de narcissisme, en raison de leurs caractéristiques de personnalité particulières, ne viennent généralement pas en psychothérapie avec une bonne compréhension de leurs problèmes ou des origines de leurs problèmes. Plus souvent, ces individus arrivent en traitement, amenés par des proches qui subissent les conséquences de leurs attitudes et de leurs conduites autocentrées. Ils peuvent demander une aide professionnelle pour les raisons suivantes :

- Un manque de reflet en miroir (les autres ne leur renvoient pas ce à quoi ils s'attendent)
- Problèmes conjugaux ou d'intimité

- Problèmes professionnels (généralement avec des collègues ou des salariés)

Les proches du patient désigné, souffrant de leur égoïsme narcissique, de leur exploitation ou de leur défaut d'empathie, "envoient " parfois leur conjoint ou membre de la famille en psychothérapie pour qu'il soit amélioré ou " réparé ».

De nombreux patients avec ces caractéristiques narcissiques ne feront appel à une aide professionnelle qu'après une collision violente avec la réalité (Mosquera, 2012) qui provoque parfois une dépression. Ceci se produit généralement quand quelque chose arrive qui rend l'image idéale intenable, qui " fait éclater la bulle »: une blessure narcissique (Knipe, 1998).

\section{La psychothérapie EMDR pour les patients atteints de narcissisme}

Dans l'approche EMDR de la psychothérapie, les problèmes psychologiques sont considérés principalement comme étant causés par l'effet cumulé des expériences traumatiques et défavorables non résolues (Shapiro, 2001, 2007). La psychothérapie EMDR a montré qu'elle constituait un traitement efficace, efficient et bien toléré de l'état de stress post-traumatique (ESPT ; Bisson \& Andrew, 2007 ; Foa, Keane, Friedman \& Cohen, 2009 ; Ursano et coll., 2004) et qu'elle est efficace pour l'ESPT déclaré à l'âge adulte ou dans l'enfance (Adler-Tapia \& Settle, 2009 ; Field \& Cottrell, 2011 ; Korn, 2009 ; Van der Kolk et coll., 2007).

Dans la psychothérapie EMDR, le patient est invité à remarquer en pleine conscience ce qui arrive aux représentations d'images visuelles perturbantes ou dysfonctionnelles, pensées négatives autoréférencées, émotions, sensations, impulsions d'agir et énoncés sur soi, tout en prêtant attention à une série de stimulations bilatérales (SBL) impliquant des mouvements oculaires alternés, des tapotements ou des sons (Leeds, 2009 ; Shapiro, 2001). L'EMDR utilise une approche en huit phases et aborde les souvenirs passés, les déclencheurs actuels et les situations futures. Pour évaluer les changements pendant le retraitement, deux échelles sont employées : l'échelle des unités subjectives de perturbation (Subjective Units of Disturbance [SUD]) et l'échelle de validité de la cognition (Validity of Cognition [VOC]). Dans les deux premières phases, le psychothérapeute recueille l'histoire du patient, développe l'alliance thérapeutique et prépare le patient à la phase de retraitement. Les phases 3 à 7 se centrent sur le retraitement d'une expérience ciblée. Dans la phase 3, le patient identifie 
une image représentative de la cible, les énoncés sur soi négatifs et positifs associés, les émotions et les sensations physiques. Le patient évalue la validité de la cognition positive (VOC : $1=$ pas vrai, $7=$ complètement vrai) et la perturbation subjective de l'émotion (SUD : $0=$ aucune perturbation, $10=$ la pire perturbation possible). Pendant la phase 4 , le patient remarque comment des éléments changent pendant qu'il prête attention aux stimulations bilatérales alternées (généralement des mouvements oculaires). La phase 4 se termine lorsque le niveau de perturbation (SUD) atteint 0 . Dans la phase 5, les stimulations bilatérales alternées sont appliquées pour renforcer le lien entre l'expérience et l'énoncé positif jusqu'à ce que le VOC atteigne 7. Dans la phase 6, les patients rapportent toute sensation résiduelle qui aurait besoin d'être retraitée. Dans la phase 7, le clincien s'assure que le patient atteint une clôture. Les patients sont invités à prendre des notes internes, en comprenant ces expériences d'événements comme faisant partie du processus. Dans la phase 8 , le clinicien réévalue les effets du retraitement et vérifie le progrès du patient.

La réalisation des procédures EMDR peut être difficile quand on travaille avec des structures de personnalité complexes caractérisées par des modes défensifs rigides et des états du soi séparés. Les parties de la personnalité peuvent fonctionner de manières très séparées les unes des autres et ne pas avoir un accès pleinement conscient les unes autres aux autres ; elles peuvent également avoir du mal à atteindre une pleine " réalisation " (Van der Hart et coll., 2006) que le présent est séparé et différent du passé (P. F. Kernberg et coll., 2000). Ce défaut de réalisation peut rendre des projections erronées sur les autres plus probables, ce qui peut causer des problèmes importants dans les relations, y compris celle avec le thérapeute. Le psychothérapeute doit prêter attention à ces distortions car elles se produisent dans la relation thérapeutique.

Le plan thérapeutique : sélection et séquence des cibles

But $\boldsymbol{d u}$ traitement. L'investissement dans une image de soi exagérément positive peut dominer le sens de soi d'une personne ou peut ne constituer qu'un état d'esprit qui émerge à différents degrés dans certaines circonstances données. Que l'investissement soit important ou limité, il peut y avoir une évolution graduelle dans la psychothérapie depuis un investissement émotionnel dans le «faux soi » vers la capacité à se sentir à l'aise en étant simplement soi-même, son "soi véritable ». L’illusion est abandonnée et cette perte comporte une compensation : un sentiment accru d'ancrage et de cohérence avec soi-même ainsi qu'une ouverture à de véritables liens avec les autres. Une personne peut arriver à accepter : "Je ne suis pas formidable, et ça va, et c'est un soulagement de savoir que je ne suis pas affreux. Je peux être simplement moi. » Il est satisfaisant pour l'individu de découvrir la permission d'être simplement humain et d'être conscient de l'humanité des autres. La psychothérapie avec ces patients est nécessairement lente et graduelle.

Cibles initiales. Masterson (1981) recommande de commencer le traitement d'un individu aux traits narcissiques en "reflétant la vulnérabilité narcissique ». En d'autres termes, la construction initiale de la relation se produit lorsque la psychothérapie reflète avec empathie la perturbation éprouvée par le patient en lien avec les moments où le faux soi n'était pas soutenu par les autres ou se révélait comme égoïste, irréaliste, gênant ou inapproprié d'une manière ou d'une autre. Par exemple, le psychothérapeute peut dire : "Je comprends que quand c’est arrivé, c’était très perturbant. Pouvez-vous m'en dire un peu plus de ce que vous avez éprouvé face à cette situation ? " De manière cohérente avec cette approche, un praticien EMDR pourrait suggérer que le ciblage démarre par des événements qui comportent une perturbation plus accessible et ceux que le patient peut facilement identifier comme négatifs et personnellement problématiques, comme des déclencheurs actuels ou des événements récents. Une blessure narcissique récente (par exemple, un événement qui a fortement miné le faux soi exagérément positif et biaisé) peut constituer une cible très accessible comportant des sentiments d'humiliation, de colère impuissante ou d'autres affects négatifs. Cette situation pourrait offrir l'opportunité de permettre au patient de se familiariser avec les procédures du traitement EMDR et d'expliquer les bénéfices de cette approche psychothérapeutique. Généralement, plusieurs situations de ce type sont disponibles dans le discours initial de la personne aux traits narcissiques car l'égocentrisme irréaliste, l'autocongratulation et le sentiment d'ayant-droit tendent à provoquer des réactions négatives chez les autres. L'auto-idéalisation irréaliste, se produisant à un degré plus ou moins important, finira le plus souvent par provoquer l'humiliation - une " collision avec la réalité ».

Cibler les premières blessures narcissiques, les pires et les récentes. Si le patient fait suffisamment confiance au psychothérapeute, de telles situations douloureuses peuvent être révélées et discutées, et si possible, les premiers exemples de cette forme particulière de 
blessure narcissique pourront être identifiés, ainsi que les exemples les pires et les plus récents. Ces situations de blessure narcissique ne permettront peut-être pas d'accéder directement aux expériences fondamentales de la petite enfance, mais elles sont beaucoup plus susceptibles d'être abordées avec une sécurité émotionnelle et moins de risque pour la stabilité de la relation thérapeutique. Plusieurs de ces situations étant abordées et traitées avec succès au fil du temps, l'alliance thérapeutique s'en trouvera renforcée, conduisant le patient à devenir capable de travailler directement sur les expériences fondamentales de son enfance, des expériences de désinvestissement parental, d'abandon émotionnel ou de maltraitances.

Pour certains patients, il sera utile de présenter ce type de travail comme étant une procédure de «soulagement du stress ». Par exemple, les patients peuvent venir en psychothérapie en se plaignant d'une personne qui «s'est mal comportée », générant du stress. Le psychothérapeute peut dire : "Ceci pourrait permettre que cette situation ne vous ennuie pas autant, même si cela n'enlèvera pas nécessairement tout le stress que vous éprouvez. »

À mesure que le stress de ces blessures narcissiques diminue et que le patient devient capable de se rappeler ces situations avec plus de clarté et moins de perturbation, un changement subtil mais important peut avoir lieu - une évolution depuis la tendance à se centrer sur ses propres besoins, sur son propre sentiment d'ayant-droit, vers une capacité à voir les autres non pas comme des objets mais comme des personnes réalles et distinctes. L'empathie - l'intérêt et l'implication envers les besoins distincts et les expériences des autres - peut être présentée au patient comme une solution à un problème jusquelà déroutant : "Pourquoi les autres ne réagissent-ils pas positivement à mon égard alors que je suis si impressionnant?»

Peu à peu, le patient aux traits narcisiques peut arriver à voir et à sentir l'importance de l'empathie envers les autres et à quel point il s'agit de la clé pour bien s'entendre avec les autres. Quand cela arrive, le patient peut aussi faire l'expérience d'une modification de la manière même dont il se définit en une perception de soi plus réaliste et équilibrée. Un patient a dit : «Je vois maintenant que je ne suis pas si bon, ni si mauvais!»

Cibler les événements positifs initiaux qui ont créé l'auto-idéalisation. Quand le patient commence à être capable d'avoir ce genre de prise de conscience accompagnée d'un ressenti, la porte peut s'ouvrir permettant de revenir en arrière et de cibler spécifiquement les événements positifs qui ont créé l'auto-idéalisation au départ, en ciblant spécifiquement l'affect positif associé à ces événements. Il peut s'agir de souvenirs où les parents ont cédé aux caprices du patient, de situations actuelles dans lesquelles l'individu satisfait un besoin obsessionnel d'obtenir l'admiration des parents dans le monde présent, ou d'une fois où le patient a pu susciter l'admiration d'autres personnes. Le plus souvent, quand ces moments sont ciblés, la visée défensive de l'auto-idéalisation devient plus claire, c'est-à-dire qu'il devient apparent que le concept de soi excessivement positif constituait une façon d'éviter ou de ne pas éprouver la douleur émotionnelle des négligences ou des événements traumatiques. Inversement, il peut être utile d'identifier et de cibler des moments dans le passé où s'est produite une blessure narcissique - quelque chose est arrivé et a rendu le concept de soi narcissique intenable et la personne s'est sentie humiliée et couverte de honte, au moins pendant une courte durée, jusqu’à la restauration du concept de soi parfait. Ce sont tous des exemples de ciblage de souvenirs comportant un affect positif stocké de manière dysfonctionnelle, des souvenirs qui ont jeté les bases des défenses d'idéalisation qui empêchent le plein accès à des souvenirs négatifs de maltraitance ou de défaut d'investissement de la part des parents. Généralement, ce n'est qu'après avoir travaillé sur plusieurs exemples de la manière dont les défenses d'idéalisation ont commencé, et lorsque l'alliance est forte et que le patient a acquis un sentiment de maîtrise dans le travail sur des souvenirs inconfortables, que nous pouvons passer au retraitement des souvenirs traumatiques fondamentaux (Mosquera, 2012).

Scénarios futurs. Les scénarios futurs devraient être utilisés pour compléter l'approche en trois volets et consolider les résultats obtenus après le traitement des souvenirs passés et des déclencheurs actuels.

\section{La phase 1 dans la psychothérapie EMDR avec} des individus atteints de narcissisme : recueil de l'histoire

Pendant la phase 1, il sera particulièrement nécessaire d'établir une connexion thérapeutique forte par le biais d'une compréhension empathique, juste et répétée de la part du psychothérapeute. Nous voulons que le patient ait le sentiment clair : "Mon thérapeute comprend vraiment ! Il comprend qui je suis ! " Le rôle du psychothérapeute consiste à aider le patient atteint de narcissisme à développer une curiosité quant aux raisons pour lesquelles les autres réagissent souvent négativement et ainsi à commencer à s'apercevoir 
que son concept de soi exagéré est irréaliste et voué à l'échec. Il importe, dans la discussion des notions de supériorité et d'ayant-droit du patient, que le psychothérapeute emploie des mots qui expriment une sensibilité vis-à-vis de la vulnérabilité sous-jacente et des défenses d'auto-idéalisation du patient, afin de s'assurer que des sentiments de honte ou d'attaque ne surgissent pas (Mosquera, 2012). Le faux soi supérieur, avec son sentiment d'ayant-droit (Kohut, 1971), bien qu'il apparaisse comme fort et robuste, est typiquement très fragile, un vernis très fin qui recouvre des sentiments de vide et souvent d'énormes doutes à sa propre encontre. Le patient peut se montrer " grossier ", sans manifester de conscience des implications insultantes de ce qu'il dit ou des effets d'une certaine déclaration sur une autre personne. Lors des premières séances, il serait probablement préférable que le psychothérapeute évite de réagir à cette grossièreté par des interprétations prématurées. Dans certains cas, même des attaques verbales contre le psychothérapeute ne nécessitent qu'une réponse minimale - ces attaques peuvent constituer un " test " motivé inconsciemment pour vérifier si le thérapeute est capable de maintenir son estime de soi (c'est-à-dire que le patient a besoin de savoir si le psychothérapeute possède un sens de soi suffisamment résilient) face aux attaques. Le patient peut être surpris thérapeutiquement par cette résilience chez le psychothérapeute - une résilience qui peut se trouver fortement en contraste avec la réponse agacée jusque-là reçue d'autres personnes.

Les patients ne tireront pas de bénéfice de la psychothérapie s'ils l'abandonnent, c'est pourquoi les psychothérapeutes doivent rester attentifs aux dangers associés à la confrontation prématurée des conduites de "passage à l'acte ". Un certain niveau de relation thérapeutique positive est nécessaire pour que la confrontation soit efficace; plus la confrontation est importante, plus la relation doit être forte. Quand le patient sait que son psychothérapeute « est de son côté » et qu'il agit dans son meilleur intérêt, alors la confrontation, exprimée avec compassion et empathie, sera plus efficace. En d'autres termes, le psychothérapeute doit veiller à ne pas confronter le matériel du patient lors des toutes premières séances, quel que soit le degré d'égoïsme apparent (sauf, évidemment, si le comportement du patient est sur le point d'avoir des effets très délétères). Les premières séances peuvent être remplies de défenses et ceci peut continuer pendant de nombreuses séances parce que le patient a besoin de se prouver encore et encore que le psychothérapeute ne va pas riposter comme l'ont fait beaucoup d'autres personnes dans la vie du patient. Cela ne signifie pas que le thérapeute doive être faux, mais simplement sensible au risque que le patient interprète mal, comprenne réellement mal tout commentaire négatif. Paradoxalement, cette approche peut ensuite avoir pour effet que le patient devienne plus détendu et prêt à dévoiler des aspects de lui-même potentiellement vulnérables.

\section{La phase 2 de la psychothérapie EMDR : la préparation}

Au cours de la phase 2 de la psychothérapie EMDR, le clinicien devrait éviter de formuler des phrases qui sous-entendraient qu'il considère que le patient aura besoin d'une stabilisation prolongée car cela pourrait provoquer l'activation de défenses et interférer avec le traitement.

Les interventions psychoéducatives. La phase de préparation sera un processus long et patient de démantèlement des nombreuses couches défensives présentes chez les partients qui présentent des traits narcissiques. La psychoéducation est très souvent utile pour décrire comment les défenses peuvent constituer une réponse adaptative à un environnement difficile dans l'enfance. Les défenses sont expliquées en tant que tentative d'aider le soi, pas en tant que défaillance personnelle ou échec. Les traumas survenus tôt dans la vie peuvent être identifiés mais, étant donnée la fragilité de la structure de personnalité initiale du patient, il ne serait pas opportun de commencer par le retraitement des pires épisodes ou traumas car il y a d'abord plusieurs obstacles à surmonter avant d'y parvenir (Mosquera, 2012).

La psychoéducation et la réflexion collaborative, ainsi que la reformulation des problèmes et de l'histoire du patient, sont nécessaires avant d'identifier des souvenirs pertinents enregistrés de manière dysfonctionnelle qui alimentent la structure narcissique. Après ce processus psychoéducatif, les patients sont capables d'un raisonnement très différent sur leur situation. Voyons quelques exemples (Mosquera, 2008) :

Pierre : Mes parents m'ont toujours traité comme un être d'exception, je croyais que j'étais unique, supérieur . . . l'entrée dans le monde réel a été très traumatisant pour moi. Je me demandais pourquoi ils (les autres) ne réalisaient pas à quel point j'étais formidable. Avec le temps, j'ai réalisé que c'était moi qui n'y trouvais pas ma place et puis, si j'étais si exceptionnel, pourquoi mes relations ne fonctionnaient-elles pas ? Pourquoi j’avais toujours des problèmes avec les autres? Mon atttitude arrogante n'aidait en rien mais je n'en étais pas conscient et je méprisais les autres parce 
qu'ils n'étaient pas capables de reconnaître ma valeur ... j'avais tellement tort ... je n'ai jamais envisagé la possibilité que mon attitude puisse influencer tout le reste, je pensais que je méritais de recevoir un traitement de faveur simplement parce que j'étais moi, quelle honte ... (p. 8)

Susanne : Maintenant, avec le recul, je pense que mes parents m'ont aimée tendrement mais m’ont fait énormément de mal. Je ne le dis pas avec ressentiment, comme c'était le cas avant, mais comme quelque chose qui est réel, quelque chose qu'ils n'ont pas pu m'offrir dans mon éducation, les principes fondamentaux des relations avec les autres, l'importance de prendre les autres en considération, et comme il peut être enrichissant d'apprendre à connaître d'autres points de vue. Je n'y aurais jamais pensé auparavant. (p. 8)

Jean : J'ai toujours éprouvé du mépris envers les autres, leurs intérêts, leurs opinions, leurs critères ... considérant toujours qu'ils n'atteignaient pas mon niveau de conversation ... quelle absurdité ... j’ai passé des années à alimenter la colère et le sentiment d'injustice, à la recherche de chaque détail qui pouvait justifier ma position ... en me comparant toujours aux autres. J'ai le sentiment d'avoir gâché de nombreuses opportunités depuis tout ce temps ... rien n'était assez bien pour moi, tout travail me paraissait «trop petit » pour moi ... si le salaire n'était pas trop bas, alors c'était le statut, ou sinon mes collègues et mes chefs étaient incompétents, ou alors, ils ne me valorisaient pas et ne me traitaient de la manière que je "méritais ». J’ai laissé passer de nombreuses opportunités mais ce qui est différent maintenant, c'est que sens que c'est moi qui ai perdu ces opportunités, elles ne m’ont pas été " volées », je n’ai pas été saboté ... avant je pensais que les autres avaient un problème, maintenant $j$ 'ai conscience que le problème était en moi ... (p. 8)

L'introduction des stimulations bilatérales dans la phase 2 pour les patients atteints de narcissisme. Les stimulations bilatérales peuvent être utilisées dans la phase 2 pour renforcer certaines interventions et pour traiter des cibles non traumatiques comme des défenses ou des problèmes relationnels. Le point essentiel à ce stade est de ne pas se laisser tromper par la sécurité, la stabilité et le contrôle apparents du patient ni de surestimer la résilience du patient. La fragilité cachée peut être importante et le patient peut s'effondrer si on traite des défenses qui sont encore nécessaires pour maintenir un sentiment de stabilité et de contrôle.
Stratégies de stabilisation. Les patients qui présentent des traits narcissiques doivent apprendre à développer des capacités de régulation émotionnelle ou de relations interpersonnelles. Cette information doit être abordée avec prudence car ils peuvent être hypersensibles à tout message suggérant qu'ils ne sont pas supérieurs, capables ou compétents en tout. Un travail sur l'autodifférenciation peut être nécessaire comme pour les autres troubles de la personnalité (Mosquera, 2012 ; Mosquera \& Gonzalez, 2014).

Même des interventions qui sont généralement stabilisatrices, comme l'installation de ressources (Leeds, 2009) ou le travail sur les manières habituelles de prendre soin de soi (Gonzalez \& Mosquera, 2012 ; Mosquera \& Gonzalez, 2014), ne devraient pas être employées avant que le patient soit capable de progresser au niveau de la réflexion et d'atteindre un sens de soi plus réaliste. Quand le patient fait preuve d'une évaluation objective et juste de ses problèmes, alors des ressources saines et des habitudes de prise de soin de soi peuvent être installées.

Le travail sur des souvenirs positifs idéalisés. Le travail sur les défenses (Knipe, 1998, 2005, 2014), ciblant des souvenirs aux affects positifs en lien avec le faux soi peut être utile pour un patient qui est devenu conscient qu'une idéalisation déformée de soi est vouée à l'échec. Par exemple, un patient de 31 ans était troublé par le fait que sa compagne n'était pas suffisamment impressionnée par ses revenus élevés. Parfois, il était désemparé par sa déclaration que le niveau de ses revenus lui importait peu et à d'autres moments, il interprétait ses paroles comme signifiant qu'elle ne l'aimait pas. Il lui a été utile de revenir à des souvenirs très précis de la manière dont ses parents lui avaient transmis l'idée que la capacité à gagner beaucoup d'argent signifie qu'on a de la valeur. Ce message avait été formulé dans le contexte d'un manque d'implication des parents envers lui et même de critiques sévères occasionnelles. Ainsi il avait appris que " pour être proche d'une autre personne, il faut gagner beaucoup d'argent et donc être considéré comme quelqu'un de valeur. Si on ne reconnaît pas mon niveau de revenus, cela signifie que je laisse la personne indifférente. " Il lui a été utile de cibler le sentiment positif associé à un souvenir précis - une occasion où il a informé ses parents de son salaire important. L'affect positif pour ce souvenir, initialement un " 10 » (sur une échelle de 0 à 10), a diminué avec plusieurs séries de stimulations bilatérales, et ce résultat s'accompagnait de plusieurs images de solitude dans l'enfance. Quand celles-ci ont été ciblées, à l'aide des phases 3 à 7 de l'EMDR, cet homme a atteint un état de résolution et une nouvelle appréciation du message aimant d'acceptation que 
sa compagne tentait de lui faire comprendre. Cette approche, dite méthode du niveau d'affect positif (LOPA : level of positive affect ; Knipe, 1998, 2014) ne devrait être utilisée avec ces patients que quand une relation thérapeutique suffisamment forte a été établie parce qu'elle implique pour le patient un niveau élevé de divulgation de soi. Un psychothérapeute expérimenté pourrait penser : «Est-ce une bonne idée de réduire la force d'une défense psychologique ? Cela ne laissera-t-il pas le patient encore plus vulnérable aux souvenirs et sentiments post-traumatiques perturbants ? "Selon notre expérience, le plus souvent, ce n'est pas le cas. Lorsque les origines du faux soi idéalisé du patient sont ciblées, dans le contexte d'une relation thérapeutique solidement établie, la force de la défense narcissique tend à diminuer, avec une réduction parallèle de l'intensité du matériel mnésique traumatique sous-jacent. Ainsi, le matériel peut être ciblé en toute sécurité à l'aide des procédures EMDR standard pour les souvenirs perturbants.

L'idéalisation peut concerner la personne ellemême ou les autres. Par exemple, une patiente se trouvait en danger à cause de son attitude idéalisatrice concernant sa relation avec un petit ami problématique. Alors qu'il se trouvait en prison après avoir tenté de la tuer, elle était dans le déni de la situation et s'accrochait aux moments idéalisés partagés avec lui. En ciblant le meilleur moment idéalisé, elle a pu prendre conscience de la situation dans laquelle elle se trouvait.

Travailler avec des parties et la dissociation. Si l'environnement familial était extrêmement négligeant, indifférent ou maltraitant, il peut y avoir des divisions prononcées au sein de la structure de la personnalité (Afifi et coll., 2011 ; Bennett, 2006 ; Lyons-Ruth, Dutra, Schuder \& Bianchi, 2006 ; Mosquera, 2012 ; Van der Hart et coll., 2006). Il peut y avoir une séparation amnésique entre l'état du soi positif idéalisé et d'autres états du soi qui contiennent les souvenirs d'abandon et de solitude, ou de violences.

La compréhension des patients atteints de narcissisme comme ayant différents états du soi ou parties (par exemple, un faux soi grandiose, un enfant vulnérable, une partie protectrice agressive) peut aider le patient à comprendre la structure sous-jacente et à planifier les interventions. Le patient peut avoir accès consciemment à ces parties ou à des souvenirs qui activent ces parties, et si c'est le cas et que le patient peut obtenir une sécurité émotionnelle suffisante avec le psychothérapeute, alors ces souvenirs peuvent être ciblés à l'aide des procédures EMDR standard.

La séquence des cibles devrait être considérée en fonction du besoin actuel du patient en termes de sécurité émotionnelle et de stabilité, mais aussi du contrat thérapeutique. Mais s'il y a un trouble dissociatif comorbide, c'est-à-dire une histoire d'attachement désorganisé dans l'enfance et une séparation dissociative entre parties de la personnalité, le déroulement du traitement devra tenir compte d'une " approche progressive " du travail avec les phobies dissociatives et avec le système interne des parties (Gonzalez \& Mosquera, 2012 ; Van der Hart, Groenendijk, Gonzalez, Mosquera \& Solomon, 2013).

\section{La phase 3 : évaluation}

Pendant la phase 3, plusieurs problèmes particuliers peuvent surgir dans le travail avec cette population. Il apparait que les patients qui ont un style narcissique n'aiment pas qu'on leur demande d'évaluer leurs expériences de manière chiffrée, comme c'est le cas avec le VOC et le SUD, peut-être parce que les chiffres obligent les patients à regarder de près leur expérience intérieure.

Les problèmes de la phase 3 sont habituellement liés aux cognitions. Lors des premiers ciblages, le psychothérapeute ne devrait pas trop insister pour obtenir une cognition négative $(\mathrm{CN})$ autoréférencée. Il peut être aidant de permettre au patient d'exprimer des $\mathrm{CN}$ sur les autres (ou même de les interroger à ce sujet), avant de demander une $\mathrm{CN}$ autoréférencée. Il peut être utile d'accepter des cognitions comme « cela me dérange encore " (quelque chose de très modéré par rapport à d'autres $\mathrm{CN}$ mais que la plupart des personnes atteintes de narcissisme sont susceptibles d'accepter) (Knipe, 1998). La cognition positive (CP) dans ce cas peut être quelque chose d'également modéré : «cela ne me dérange plus». En ce qui concerne les $\mathrm{CP}$, nous devons veiller à ne pas accepter une $\mathrm{CP}$ idéalisée comme " je suis le meilleur » ou " je suis supérieur ", même si c'est quelque chose que le patient peut mentionner quand on lui demande la CP désirée (Mosquera, 2012). Une difficulté dont il faut tenir compte ici est que les questions de la phase 3 peuvent recevoir différentes réponses de différentes parties de la personnalité distinctes. Une réponse peut provenir d'une partie du patient qui porte la perturbation et une autre réponse peut émerger du faux soi idéalisé. Par exemple, un patient peut identifier un souvenir perturbant avec une $\mathrm{CN}$ « cela me dérange vraiment ! » Puis, le patient peut dire qu'une CP associée, « cela ne me dérange pas du tout », a un VOC de « sept»!

La phase 4 : désensibilisation

Les cliniciens devraient garder à l'esprit que selon le modèle TAI, nous nous attendons à ce que des séries 
centrées de stimulations bilatérales aident à relier les informations adaptatives, mais cela ne signifie pas que ces associations seront toujours vécues comme positives. Par exemple, un patient qui a une défense égocentrique consistant à toujours rejeter la faute sur les autres pourrait devenir de plus en plus conscient de sa part de faute et de responsabilité personnelle dans les événements difficiles. Par exemple, «je suis coupable » est plus adaptatif que « les autres sont à l'origine de mes problèmes » dans des situations où le patient possède réellement une part de responsabilité dans ce qui s'est passé. Le traitement peut avoir l'air d'un « traitement de renversement ", du positif (idéalisé) vers le négatif (réaliste). Cette possibilité montre pourquoi une alliance thérapeutique et une confiance solides sont si nécessaires dans le travail avec des patients aux traits narcissiques. Quand la psychothérapie se déroule d'une manière aidante et appropriée, le patient révèle forcément une fragilité et une vulnérabilité énormes. Le psychothérapeute doit être attentif à éviter les mots qui pourraient comporter une implication de honte et le désinvestissement du patient par rapport au faux soi idéalisé doit avancer à un rythme que le patient peut tolérer.

Parfois les psychothérapeutes doivent adopter une posture active pour garantir que toutes les émotions sont incluses dans la phase de désensibilisation. Par exemple, dans l'histoire du patient, la peur ou la tristesse n'étaient peut-être pas autorisées. Le patient n'avait le droit que d'être un enfant parfait idéalisé, sans les sentiments ou autres comportements que les parents ne pouvaient pas tolérer. Le psychothérapeute peut utiliser des tissages comme "permettez-vous d'éprouver TOUTES vos émotions " ou " laissez-les s'exprimer, c'est OK».

\section{La phase 5 : installation}

Certains patients ont du mal à trouver une CP car ils pensent à tort qu'ils doivent être convaincus de cette croyance avant de commencer le retraitement EMDR. Pour ces patients, le psychothérapeute peut dire : «Je ne m'attendrais pas à ce que vous ayez un score (VOC) élevé au début de ce travail. Si votre score est un 1, 2 ou 3, cela signifie simplement que nous travaillons sur ce qui doit l'être. »

Dans certains cas, cette phase peut s'avérer compliquée car les patients provenant d'environnements négligeants ou traumatiques dans la petite enfance peuvent ne pas avoir de réseaux positifs fondés sur la réalité avec lesquels faire des liens. D'autres n'ont pas connu d'expériences de partage d'affects positifs avec leurs parents dans l'enfance (Leeds, 2009). Ces patients peuvent tout simplement ne pas connaitre les satisfactions d'un engagement empathique auprès d'autres personnes parce que de telles expériences n'ont pas existé dans leur histoire personnelle ou, pire encore, l'engagement véritable avec d'autres était systématiquement puni ou ignoré.

Un " moment d'enseignement " important se produit souvent au cours de la phase 5, lorsque le patient vient à réaliser qu'une $\mathrm{CP}$ de supériorité ou d'ayant-droit n'est en fait pas réaliste ou viable. À ce moment, le psychothérapeute peut suggérer une $\mathrm{CP}$ plus appropriée. Par exemple, plutôt qu'une $\mathrm{CP}$ défensive comme « je suis intouchable/inébranlable», il peut proposer une $\mathrm{CP}$ davantage fondée sur la réalité comme «je peux me protéger».

\section{La phase 6 : scanner corporel}

Dans la phase 6, il semble y avoir moins de variations d'avec le protocole habituel. Certains patients aux traits narcissiques ont appris à dissocier certaines sensations corporelles et celles-ci peuvent être davantage disponibles à la conscience après les phases EMDR précédentes. Par exemple, après les phases 4 et 5 , un patient peut rapporter plus de tension physique ou de fatigue, ou de la faim (si la dissociation de la faim faisait partie du faux soi initial, comme cela se produit parfois chez les patients anorexiques). Le psychothérapeute devrait rester attentif à la possibilité que ces sensations physiques soient le début de nouveaux canaux d'informations traumatiques et s'assurer que le patient est suffisamment capable de contenir toute information émergeante avant de quitter la séance.

\section{La phase 7 : clôture}

Il est souvent préférable de laisser une cible incomplète et de terminer la séance avec une clôture adéquate et sûre que de trop insister. Certaines cibles, tant les souvenirs d'auto-idéalisation que les souvenirs traumatiques ou de négligences, peuvent nécessiter plusieurs séances. En plus des procédures de clôture habituelles centrées sur l'apaisement des sensations de tension, les auteurs recommandent aussi de poser des questions comme " qu'est-ce que vous avez compris ici aujourd'hui qui vous soit utile? " ou « en partant aujourd'hui, de quoi voudriez-vous vous souvenir quelque chose qui vous sera vraiment utile? ».

\section{La phase 8 : réévaluation}

Les patients manifestant initialement des traits narcissiques peuvent avoir besoin de plus de temps et de séances pour consolider ce qu'ils ont compris au cours 
des séances consacrées au traitement de souvenirs. Lors d'une séance, les patients peuvent développer une compréhension claire des façons dont leur image de soi exagérément positive et idéalisée a été un obstacle à la réalisation de leurs buts les plus importants. De plus, les patients peuvent éprouver un désinvestissement émotionnel quant au sentiment recherché de supériorité ou d'ayant-droit. Néanmoins, entre les séances, ils peuvent revenir à leurs manières défensives d'être en relation avec les autres et à l'idéalisation de soi. Il peut être nécessaire, au cours de nombreuses séances de psychothérapie, d'identifier et de cibler des événements supplémentaires qui constituent les fondements de l'auto-idéalisation inappropriée. Il peut exister des souvenirs d'avoir été très gâté à l'âge de sept ans, d'avoir eu plus de facilités qu'un frère ou une sœur à l'âge de neuf ans, d'un sentiment de supériorité à l'égard de camarades de classe au collège, d'une tendance à négliger les émotions des autres dans leurs relations actuelles ou dans la sphère professionnelle, et ainsi de suite. À mesure que chacune de ces cibles dysfonctionnelles associées à des affects positifs se résout, l'investissement émotionnel de la personne en son faux soi narcissique se réduira probablement. Il sera alors généralement important d'attirer l'attention du patient sur la satisfaction accrue qui est maintenant possible dans les relations investies avec les autres, avec un engagement empathique sans le versant compétitif.

La phase de réévaluation est essentielle à un retraitement adéquat des cibles. Il importe de rester centré et d'éviter de passer continuellement d'une cible à une autre. Le patient peut évoquer différents aspects sur lesquels travailler mais il faut garder le bon cap.

Certains cas évoluent lentement, particulièrement ceux qui n'ont jamais suivi de psychothérapie auparavant et qui n'ont donc pas, au départ, de véritable identité fondamentale. Avec le temps, à mesure que nous enlevons les armures et les couches défensives, un fort sentiment de "voici qui je suis vraiment!» peut émerger.

Ces cas peuvent nécessiter un travail de reconstruction après les séances de retraitement pour que les patients apprennent à observer intérieurement, à reconnaître qui ils sont vraiment et comment fonctionner à partir de cette perspective nouvelle. Les patients peuvent sembler aller moins bien pendant ces moments où ils entrent en contact avec le soi véritable, sans les structures défensives, alors qu'il leur manque encore une véritable identité. Pendant cette partie de la psychothérapie, quand certains problèmes fondamentaux sont traités, le travail sur les habitudes pour prendre soin de soi devient pertinent en tant que repère pour reconstruire une nouvelle façon d'être en lien avec son soi véritable. Le développement et l'installation de ressources sont également importants pour créer ou renforcer les aspects adaptatifs et sains de la personnalité (par exemple, un engagement empathique envers les autres). Il est important que le psychothérapeute remarque et apprécie les changements positifs chez le patient et qu'il tisse cette reconnaissance positive avec le travail difficile consistant à renoncer à des défenses employées depuis longtemps et à cibler directement des souvenirs perturbants.

\section{Conclusion}

Les traits narcissiques peuvent être une conséquence d'un concept de soi faussé et exagérément idéalisé résultant d'un grand nombre de situations dans l'enfance, comprenant des éloges excessifs et une surprotection. De plus, ce concept de soi idéalisé est lié au besoin de créer des défenses psychologiques pour empêcher la pleine émergence de souvenirs de négligences et de maltraitances sévères. Pour qu'un processus psychothérapeutique EMDR puisse se dérouler, il faut comprendre la voie particulière que chaque patient a empruntée depuis ses expériences de début de vie particulières jusqu’à ses problèmes présents réels.

Le traitement efficace du narcissisme implique que la définition de soi du patient et la manière dont il perçoit le problème fasse écho chez les psychothérapeute ; il faut susciter chez le patient une réflexion sur soi au cours de la phase de préparation ; identifier les nombreuses déviations et impasses possibles qui peuvent résulter d'une suractivation des défenses ; identifier et traiter les expériences fondamentales de désinvestissement, de négligence et de trauma qui ont pu être recouvertes initialement (c'est-à-dire bloquées de la conscience) par les défenses ; identifier et corriger avec empathie les distortions de la perception qu'a le patient du psychothérapeute; et éviter les réactions contre-transférentielles non thérapeutiques prenant leur source chez le psychothérapeute.

\section{Bibliographie}

Adler-Tapia, R. \& Settle, C. (2009). Evidence of the efficacy of EMDR with children and adolescents in individual psychotherapy: A review of the research published in peer-reviewed journals. Journal of EMDR Practice and Research, 3(4), 232-247.

Afifi, T. O., Mather, A., Boman, J., Fleisher, W., Enns, M. W., MacMillan, H. \& Sareen, J. (2011). Childhood adversity and personality disorders: Results from a nationally representative population-based study. Journal of Psychiatric Research, 45(6), 814-822. 
American Psychiatric Association. (2013). Diagnostic and statistical manual of mental disorders (5th ed.). Arlington, VA: Author.

Battle, C. L., Shea, M. T., Johnson, D. M., Yen, S., Zlotnick, C., Zanarini, M. C. . . . Morey, L. C. (2004). Childhood maltreatment associated with adult personality disorders: Findings from the Collaborative Longitudinal Personality Disorders Study. Journal of Personality Disorders, 18, 193-211.

Bennett, S. (2006). Attachment theory and research applied to the conceptualization and treatment of pathological narcissism. Clinical Social Work Journal, 34(1), 45-60.

Bierer, L. M., Yehuda, R., Schmeidler, J., Mitropoulou, V., New, A. S., Silverman, J. M., \& Siever, L. J. (2003). Abuse and neglect in childhood: Relationship to personality disorder diagnoses. CNS Spectrum, 8, 737-754.

Bisson, J. \& Andrew, M. (2007). Psychological treatment of post-traumatic stress disorder (PTSD). Cochrane Database Systematic Review, (3), CD00338.

Cai, H., Kwan, V. \& Sedikides, C. (2012). A sociocultural approach to narcissism: The case of modern China. European Journal of Personality, 26, 529-535.

Campbell, W. K. \& Foster, C. A. (2002). Narcissism and commitment in romantic relationships: An investment model analysis. Personality and Social Psychology Bulletin, 28, 484-494.

Cohen, L. J., Tanis, T., Bhattacharjee, R., Nesci, C., Halmi, W. \& Galynker, I. (2014). Are there differential relationships between different types of childhood maltreatment and different types of adult personality pathology? Psychiatry Research, 215(1), 192-201.

Collishaw, S., Pickles, A., Messer, J., Rutter, M., Shearer, C. \& Maughan, B. (2007). Resilience to adult psychopathology following childhood maltreatment: Evidence from a community sample. Child Abuse and Neglect, 31, 211-229.

Cramer, P. (2010). Young adult narcissism: A 20-year longitudinal study of the contribution of parenting styles, preschool precursors of narcissism, and denial. Journal of Research in Personality, 45(1), 19-28.

DeWall, C. N., Pond, R. S., Campbell, W. K. \& Twenge, J. M. (2011). Tuning in to psychological change: Linguistic markers of psychological traits and emotions over time in popular U.S. song lyrics. Psychology of Aesthetics, Creativity, and the Arts, 5, 200-207.

Field, A., \& Cottrell, D. (2011). Eye movement desensitization and reprocessing as a therapeutic intervention for traumatized children and adolescents: A systematic review of the evidence for family therapists. Journal of Family Therapy, 33(4), 374-388.

Foa, E., Keane, T., Friedman, M. \& Cohen, J. (Eds.). (2009). Effective treatments for PTSD: Practice guidelines from the International Society for Traumatic Stress Studies. New York, NY: Guilford Press.

Foster, J. D., Campbell, W. K. \& Twenge, J. M. (2003). Individual differences in narcissism: Inflated self-views across the lifespan and around the world. Journal of Research in Personality, 37, 469-486.
Gabbard, G. (1989). Two subtypes of narcissistic personality disorder. Bulletin of the Menninger Clinic, 53, 527-532.

Gibb, B. E., Wheeler, R., Alloy, L. B., \& Abramson, L. Y. (2001). Emotional, physical, and sexual maltreatment in childhood versus adolescence and personality dysfunction in young adulthood. Journal of Personality Disorders, $15,505-511$.

Gonzalez, A. \& Mosquera, D. (2012). EMDR and dissociation. The progressive approach. Madrid, Spain: Ediciones Pléyades.

Grover, K. E., Carpenter, L. L., Price, L. H., Gagne, G. G., Mello, A. F., Mello, M. F. \& Tyrka, A. R. (2007). The relationship between childhood abuse and adult personality disorder symptoms. Journal of Personality Disorders, 21, 442-447.

Hamamura, T. (2012). Are cultures becoming individualistic? A cross-temporal comparison of individualismcollectivism in the United States and Japan. Personality and Social Psychology Review, 16, 3-24.

Johnson, D. M., Shehan, T. C., \& Chard, K. M. (2004). Personality disorders, coping strategies, and posttraumatic stress disorder in women with histories of childhood sexual abuse. Journal of Child Sexual Abuse, 12, 19-39.

Johnson, J. G., Cohen, P., Brown, J., Smailes, E. M. \& Bernstein, D. P. (1999). Childhood maltreatment increases risk for personality disorders during early adulthood. Archives of General Psychiatry, 56, 600-608.

Johnson, J. G., Cohen, P., Chen, H., Kasen, S. \& Brook, J. S. (2006). Parenting behaviors associated with risk for offspring personality disorder during adulthood. Archives of General Psychiatry, 63, 579-587.

Johnson, J. G., Smailes, E. M., Cohen, P., Brown, J. \& Bernstein, D. P. (2000). Association between four types of childhood neglect and personality disorder symptoms during adolescence and early adulthood: Findings of a community-based longitudinal study. Journal of Personality Disorders, 14, 171-187.

Jonason, P. K., Lyons, M. \& Bethell, E. (2014). The making of Darth Vader: Parent-child care and the Dark Triad. Personality and Individual Differences, 67(2014), 30-34.

Kernberg, O. (1975). Borderline conditions and pathological narcissism. New York, NY: Aronson.

Kernberg, P. F. (1998). Developmental aspects of normal and pathological narcissism. In E. F. Ronningstam (Ed.), Disorders of narcissism: Diagnostic, clinical and empirical implications (pp. 103-120). Washington, DC: American Psychiatric Association.

Kernberg, P. F., Weiner, A. S. \& Bardenstein, K. K. (2000). Personality disorders in children and adolescents. New York, NY: Basic Books.

Knipe, J. (1998). It was a golden time: Healing narcissistic vulnerability. In P. Manfield (Ed.), Extending EMDR (pp. 232-255). New York, NY: Norton.

Knipe, J. (2005). Targeting positive affect to clear the pain of unrequited love: Codependence, avoidance and 
procrastination. In R. Shapiro (Ed.), EMDR solutions (pp. 189-211). New York, NY: Norton.

Knipe, J. (2007). Loving eyes: Procedures to therapeutically reverse dissociative processes while preserving emotional safety. In C. Forgash \& M. Copeley (Eds.), Healing the heart of trauma and dissociation (pp. 181-226). New York, NY: Springer Publishing.

Knipe, J. (2014). EMDR toolbox: Theory and treatment for complex PTSD and dissociation. New York, NY: Springer Publishing.

Kohut, H. (1971). The analysis of the self: A systematic approach to the psychoanalytic treatment of narcissistic personality disorder. New York, NY: University of Chicago Press.

Konrath, S. H., O’Brien, E. H. \& Hsing, C. (2011). Changes in dispositional empathy in American college students over time: A meta-analysis. Personality and Social Psychology Review, 15, 180-198.

Korn, D. (2009). EMDR and the treatment of complex PTSD: A review. Journal of EMDR Practice and Research, 3(4), 264-278.

Kowalski, R. M. (2001). Behaving badly: Aversive behaviors in interpersonal relationships. Washington, DC: American Psychological Association.

Leeds, A. M. (2009). A guide to the standard EMDR protocols for clinicians, supervisors, and consultants. New York, NY: Springer Publishing.

Livesley, W. J., Jang, K. L., Jackson, D. N. \& Vernon, P. A. (1993). Genetic and environmental contributions to dimensions of personality disorder. American Journal of Psychiatry, 150(12), 1826-1831.

Lobbestael, J., Arntz, A. \& Berstein, D. P. (2010). Disentangling the relationship between different types of childhood maltreatment and personality disorders. Journal of Personality Disorders, 24, 285-295.

Luntz, B. K., \& Widom, C. (1994). Antisocial personality disorder in abused and neglected children grown up. The American Journal of Psychiatry, 151, 670-674.

Lyons, M., Morgan, K., Thomas, J. \& Al Hashmi, A. (2013). Patterns of parental warmth, attachment, and narcissism in young women in United Arab Emirates and the United Kingdom. Individual Differences Research, 11(4), 149-158.

Lyons-Ruth, K., Dutra, L., Schuder, M. R. \& Bianchi, I. (2006). From infant attachment disorganization to adult dissociation: Relational adaptations or traumatic experiences? Psychiatric Clinics of North America, 29(1), 63-viii.

Masterson, J. F. (1981) The narcissistic and borderline disorders. An integrated developmental approach. New York, NY: Routledge.

Millon, T. (1990). The disorders of personality. In L. A. Pervin (Ed.), Handbook of personality (pp. 339-370). New York, NY: Guilford Press.

Millon, T. (1996). Disorders of personality: DSM-IV and beyond (2nd ed.). New York, NY: Wiley.

Mosquera, D. (2007). Desmontando corazas. El trastorno antisocial aprendido: Un mecanismo de defensa extremo. [Removing shields: The antisocial learned: A defense mechanism end] Madrid, Spain: Ediciones Pléyades.
Mosquera, D. (2008). Personalidades narcisistas y personalidades con rasgos narcisistas. Revista Persona, 8(2). Buenos Aires, Argentina: Instituto Argentino para el Estudio de la Personalidad y sus Desórdenes.

Mosquera, D. (2012). Narcissistic personalities, trauma and EMDR. Paper presented at the National EMDR Congress, The Netherlands.

Mosquera, D. (2013, June). Understanding and treating narcissistic personality disorder with EMDR. Paper presented at the 14th EMDR Europe Association Conference, Geneva, Switzerland.

Mosquera, D. \& Gonzalez, A. (2011). Narcissism as a consequence of trauma and early experiences. ESTD Newsletter, 1(2), 4-6.

Mosquera, D. \& Gonzalez, A. (2014). Borderline personality disorder and EMDR therapy. Madrid, Spain: Ediciones Pléyades.

Nathanson, D. L. (1992). Shame and pride: Affect, sex, and the birth of the self. New York, NY: Norton.

Reich, W. (1949). Character analysis (3rd ed.). New York, NY: Farrar, Straus, \& Giroux.

Rettew, D. C., Zanarini, M. C., Yen, S., Grilo, C. M., Skodol, E., Shea T. . . Gunderson, J. G. (2003). Childhood antecedents of avoidant personality disorder: A retrospective study. Journal of the American Academy of Child and Adolescent Psychiatry, 42, 1122-1130.

Segrin, C., Woszidlo, A., Givertz, M., \& Montgomery, N. (2013). Parent and child traits associated with overparenting. Journal of Social and Clinical Psychology, 32(6), 569-595.

Shapiro, F. (2001). Eye movement desensitization and reprocessing. Basic principles, protocols and procedures (2nd ed.). New York, NY: Guilford Press.

Shapiro, F. (Ed.). (2007). EMDR as an integrative psychotherapy approach (2nd ed.). Washington, DC: American Psychological Association.

Stone, M. H. (1993). Abnormalities of personality: Within and beyond the realm of treatment. New York, NY: Norton.

Tartakoff, H. H. (1966). The normal personality in our culture and the Nobel Prize complex. In R. M. Loewenstein, L. M. Newman, M. Schur \& A. J. Solint (Eds.), Psychoanalysis-A General psychology (pp. 222-252). New York, NY: International Universities Press.

Trzesniewski, K. H., Donnellan, M. B. \& Robins, R. W. (2008). Do today's young people really think they are so extraordinary? An examination of secular trends in narcissism and self-enhancement. Psychological Science, 19, 181-188.

Twenge, J. M., Campbell, W. K. \& Gentile, B. (2012). Increases in individualistic words and phrases in American books, 1960-2008. PLoS ONE, 7, e40181.

Twenge, J. M., Konrath, S., Foster, J. D., Campbell, W. K. \& Bushman, B. J. (2008). Egos inflating over time: A crosstemporal meta-analysis of the Narcissistic Personality Inventory. Journal of Personality, 76, 875-902.

Tyrka, A. R., Wyche, M. C., Kelly, M. M., Price, L. H. \& Carpenter, L. L. (2009). Childhood maltreatment and 
adult personality disorder symptoms: Influence of maltreatment type. Psychiatry Research, 165(3), 281-287.

Ursano, R. J., Bell, C., Eth, S., Friedman, M., Norwood, A., Pfefferbaum, B. . . Y Yager, J. (2004). Practice guideline for the treatment of patients with acute stress disorder and posttraumatic stress disorder. American Journal of Psychiatry, 161(11), 3-31.

Van der Hart, O., Groenendijk, M., Gonzalez, A., Mosquera, D. \& Solomon, R. (2013). Dissociation of the personality and EMDR therapy in complex trauma-related disorders: Applications of the stabilization phase. Journal of EMDR Practice and research, 7(2), 81-94.

Van der Hart, O., Nijenhuis, E. \& Steel, K. (2006). The haunted self. Structural dissociation and the treatment of chronic traumatization. New York, NY: Norton.

Van der Kolk, B. A., Spinazzola, J., Blaustein, M. E., Hopper, J. W., Hopper, E. K., Korn, D. L. \& Simpson, W. B. (2007). A randomized clinical trial of eye movement desensitization and reprocessing (EMDR), fluoxetine, and pill placebo in the treatment of posttraumatic stress disorder: Treatment effects and long-term maintenance. Journal of Clinical Psychiatry, 68(1), 37-46.

Volkan, V. D. (1973). Transitional fantasies in the analysis of a narcissistic personality. Journal of the American Psychoanalytic Association, 21, 351-376.

Winnicott, D. W. (1965). Los procesos de maduración y el ambiente facilitador. Estudios para una teoría del desarrollo emocional. Buenos Aires, Argentina: Paidós.

Yen, S., Shea, T., Battle, C. L., Johnson, D. M., Zlotnick, C., Dolan-Sewell, R. . . McGlashan, T. H. (2002). Traumatic exposure and posttraumatic stress disorder in borderline, schizotypal, avoidant, and obsessive-compulsive personality disorders: Findings from the collaborative longitudinal personality disorders study. Journal of Nervous and Mental Disease, 190(8), 510-518.

Zanarini, M. C., Frankenburg, F. R., Reich, D. B., Marino, M. F., Lewis, R. E., Williams, A. A. \& Khera, G. S. (2000). Biparental failure in the childhood experiences of borderline patients. Journal of Personality Disorders, 14(3), 264-273.

Zanarini, M. C., Gunderson, J. G., Marino, M. F., Schwartz, E. O. \& Frankenburg, F. R. (1989). Childhood experiences of borderline patients. Comprehensive Psychiatry, 30(1), 18-25.

Zanarini, M. C., Williams, A. A., Lewis, R. E., Reich, R. B., Vera, S. C., Marino, M. F. . . Frankenburg, F. R. (1997). Reported pathological childhood experiences associated with the development of borderline personality disorder. American Journal of Psychiatry, 154(8), 1101-1106.

Zanarini, M. C., Yong, L., Frankenburg, F. R., Hennen, J., Reich, D. B., Marino, M. F. \& Vujanovik, A. A. (2002). Severity of reported childhood sexual abuse and its relationship to severity of borderline psychopathology and psychosocial impairment among borderline inpatients. Journal of Nervous and Mental Disease, 190(6), 381-387.

Merci d'adresser toute correspondance concernant cet article à Dolores Mosquera, INTRA-TP, Instituto para el estudio del Trauma y los Trastornos de la Personalidad, General Sanjurjo 111, 5 15006, A Coruña, Espagne. Courriel : doloresmosquera@gmail.com 